\title{
People, Ideas, Milestones: A Scientometric Study of Computational Thinking
}

\author{
MOHAMMED SAQR, University of Eastern Finland and KTH Royal Institute of Technology \\ KWOK NG, University of Eastern Finland and University of Limerick \\ SOLOMON SUNDAY OYELERE, Luleå University of Technology \\ MATTI TEDRE, University of Eastern Finland
}

\begin{abstract}
The momentum around computational thinking (CT) has kindled a rising wave of research initiatives and scholarly contributions seeking to capitalize on the opportunities that CT could bring. A number of literature reviews have showed a vibrant community of practitioners and a growing number of publications. However, the history and evolution of the emerging research topic, the milestone publications that have shaped its directions, and the timeline of the important developments may be better told through a quantitative, scientometric narrative. This article presents a bibliometric analysis of the drivers of the CT topic, as well as its main themes of research, international collaborations, influential authors, and seminal publications, and how authors and publications have influenced one another. The metadata of 1,874 documents were retrieved from the Scopus database using the keyword "computational thinking." The results show that CT research has been US-centric from the start, and continues to be dominated by US researchers both in volume and impact. International collaboration is relatively low, but clusters of joint research are found between, for example, a number of Nordic countries, lusophone- and hispanophone countries, and central European countries. The results show that CT features the computing's traditional tripartite disciplinary structure (design, modeling, and theory), a distinct emphasis on programming, and a strong pedagogical and educational backdrop including constructionism, self-efficacy, motivation, and teacher training.
\end{abstract}

\section{CCS Concepts: • Applied computing $\rightarrow$ Education;}

Additional Key Words and Phrases: Computational thinking, bibliometric research, history, scientometrics, literature review, computing education research, computer science education

\section{ACM Reference format:}

Mohammed Saqr, Kwok Ng, Solomon Sunday Oyelere, and Matti Tedre. 2021. People, Ideas, Milestones: A Scientometric Study of Computational Thinking. ACM Trans. Comput. Educ. 21, 3, Article 20 (February 2021), 17 pages.

https://doi.org/10.1145/3445984

\footnotetext{
Authors' addresses: M. Saqr, University of eastern Finland, School of Computing, Joensuu, Yliopistokatu 2, fi-80100 Joensuu, Finland and KTH Royal Institute of Technology, EECS - School of Electrical Engineering and Computer Science, Lindstedtsvägen 3, SE-100 44 Stockholm, Sweden; email: mohammed.saqr@uef.fi; K. Ng, University of Eastern Finland, PO Box 111, 80101 Joensuu, Finland; email: kwok.ng@uef.fi; S. S. Oyelere, Department of Computer Science, Electrical and Space Engineering, Luleå University of Technology, SE-931 87 Skellefteå, Sweden; email: solomon.oyelere@ltu.se; M. Tedre, University of eastern Finland, School of Computing, Joensuu, Yliopistokatu 2, fi-80100 Joensuu, Finland; email: matti.tedre@uef.fi.

Permission to make digital or hard copies of all or part of this work for personal or classroom use is granted without fee provided that copies are not made or distributed for profit or commercial advantage and that copies bear this notice and the full citation on the first page. Copyrights for components of this work owned by others than ACM must be honored. Abstracting with credit is permitted. To copy otherwise, or republish, to post on servers or to redistribute to lists, requires prior specific permission and/or a fee. Request permissions from permissions@acm.org.

(C) 2021 Association for Computing Machinery.

1946-6226/2021/02-ART20 \$15.00

https://doi.org/10.1145/3445984
} 


\section{INTRODUCTION}

Since the modern use of the older phrase computational thinking (CT) was introduced in 2006 [68; see also 48], a great deal has been written about the potential and importance of CT as a set of mental tools, skills, and attitudes (e.g., [6, 19, 27, 28]). Numerous definitions for the term have been proposed, with years of work invested in describing CT, its manifestations in different fields, and ways to teach it at different levels of education [22, 25, 40]. Stories of the historical evolution and emergence of the concept have been told [19]. Major organizations, including CSTA (the Computer Science Teachers' Association), CAS (Computing At School of the British Computing Society), and ACARA (Australian Curriculum, Assessment, and Reporting Authority), have developed their own frameworks for bringing CT to schools [60].

Although no consensus has formed over the definition of $\mathrm{CT}$, a frequently used quote is a description by Aho [1] of CT as "the thought processes involved in formulating problems so their solutions can be represented as computational steps and algorithms." Numerous competing definitions exist, and the term itself has been rephrased and reformulated in many ways, including computational doing [5], computational making [61], computational action [62], computational fluency [51], proceduracy [63], and computational participation [31]. It was predated by older similar ideas like algorithmic thinking [32] and procedural thinking [59]. Much of the CT discussions have been centered around how to teach computing in the K-12 setting [27], but also in higher education and in different disciplines. With all of these competing viewpoints, CT has been in the midst of a continuous back-and-forth of arguments, counter-arguments, new proposals, criticism, reviews, and analyses.

In addition to the excited rush to develop new means for teaching and evaluating $\mathrm{CT}$, there are many critical voices as well. The CT movement has been criticized for ignoring important lessons in computing education research (CER) [49, 60, 64], for either too much programming or too little programming $[3,10,11,35,39,41]$, for ambivalence between CT and computer science (CS) [24, 44], and for ambiguity on how to measure progress in CT [16]. It has been suggested that there is a spectrum of views on $\mathrm{CT}$, where at the one end are people who argue that one can learn $\mathrm{CT}$ without programming or rigorous models of computation and that computers are not necessary, and at the other end are people who argue that computers and programming are quintessential for CT $[11,16]$.

A number of excellent literature reviews and analyses of CT [22, 25, 38, 40,57] have revealed a rich body of CT literature since 2006. This article presents an alternative viewpoint: a quantitative, bibliometric analysis of the CT literature. By analyzing article metadata in Elsevier's abstract and citation database Scopus, the article establishes milestone articles, key authors, regional differences, historical growth, bibliometric trends, and a number of timelines for the emergence of CT. The article is aimed at giving the reader an overview of when and where CT developed into the flourishing topic of investigation it is today; who and what are the key publications, the most active people, and the research communities in the field; and the frequency and conceptual structure of keywords in CT.

\section{METHODOLOGY}

The data were retrieved on January 2, 2020, from Elsevier's Scopus abstract and citation database, which contains roughly 70 million records of peer-reviewed articles. The Bibliometrix R library [2] was used for analysis. The search term "computational thinking" was used for document retrieval, and only articles in English were included to enable keyword comparison. The search resulted in 1,874 documents from 595 different source titles (typically journals, books, and proceedings) (Table 1). The dataset included 3,779 authors, and among the dataset, 295 articles were single authored. 
Table 1. Basic Information About the Bibliometric Dataset

\begin{tabular}{|c|c|}
\hline \multicolumn{2}{|l|}{ Data set statistics } \\
\hline Documents & 1,874 \\
\hline Distinct source titles (journals, books, etc.) & 595 \\
\hline Keywords Plus (ID) & 5,005 \\
\hline Author Keywords (DE) & 3,138 \\
\hline Period & $2006-2019$ \\
\hline Mean citations per document & 6.828 \\
\hline \multicolumn{2}{|l|}{ Document types } \\
\hline Journal article & 473 \\
\hline Journal article (in press) & 6 \\
\hline Book chapter & 71 \\
\hline Conference paper & 1,288 \\
\hline Editorial & 11 \\
\hline Review & 25 \\
\hline \multicolumn{2}{|l|}{ Authorship data } \\
\hline Authors & 3,779 \\
\hline Author appearances & 5,914 \\
\hline Authors of single-authored documents & 295 \\
\hline Authors of multi-authored documents & 3,484 \\
\hline Single-authored documents & 348 \\
\hline Mean documents per author & 0.496 \\
\hline Mean authors per document & 2.02 \\
\hline Mean co-authors per document & 3.16 \\
\hline Mean collaboration index & 2.28 \\
\hline
\end{tabular}

The analysis was limited to the period from 2006 to 2019; as there were only three occurrences before 2006, none of them relevant. Journal articles, conference papers, reviews, book chapters, editorials, and viewpoints were included. Although editorials are typically not peer reviewed, they were included in the dataset because some of the most influential writings on the topic-including Wing's 2006 essay [68]-have been published as non-peer-reviewed editorials or viewpoints. Book chapters were included, and a number of conference proceedings are classified as edited books. The data were cleaned, duplicates were removed, and author names, journals, and conferences with different spellings were checked and fixed. The analysis included descriptive statistics of document count, document metadata, authors, sources, article types, and other measures. As "country" is not a standard publication attribute in the database, countries of authors were extracted from the first author's first affiliations. The collaboration index was computed as the ratio of the sum of authors of multi-authored articles to total multi-authored articles (Table 1). The annual percentage growth Rate was calculated as the average percentage increase in the number of articles over a period of a year.

For keywords analysis, four types of keywords were included in the study: author keywords (keywords provided by the author), categorized keywords (duplicates and similarities aggregated), keywords extracted from titles, and keywords extracted from abstracts. Using the four types of keywords helps to triangulate the conceptual structure of CT publications, and to overcome the shortcomings of author-provided keywords. Of the four types of keywords, only author keywords data were cleaned-for instance, keywords in plural were combined with singular ("primary school"/"primary schools"), and similar terms and abbreviations were combined 
(“computer science"/CS" and“K12"/“K-12"/“K-12"). The abstract keywords were extracted from the retrieved articles using the term extraction method of the Bibliometrix R library [2]; that method includes deletion of stop words and application of a stemming algorithm that reduces words to their stem. A keyword co-occurrence network was constructed to map the relationships between different concepts within each document. For readability of relationships and labels, the network sizes were limited to the 30 most frequent keywords. The analysis of keywords evolution used the cumulative frequency of keywords across the years.

The keywords were clustered using network community detection (a "community of keywords" is a group of keywords that occur frequently together) using the Louvain modularity algorithm, which has been shown to be computationally efficient with very good clustering results [13]. Each keyword community was uniquely colored in the network plot. To understand the structure of collaborating networks, the Louvain modularity algorithm for community detection was also applied to cluster countries that collaborate frequently together. Each community of countries was uniquely colored in the network plot.

The study also used historiography mapping, a popular bibliometric method developed by Garfield [23] to map the chronological direct citation network within a set of papers. The method maps the most relevant papers by ranking them by their citations within the examined datasetknown as the local citation score (LCS)-as opposed to the papers' overall citations-known as the global citation score (GCS). Papers with high LCSs are considered more relevant to the examined topic, and they show higher on the graph. For each author in the dataset, the $h$-index and its variants ( $m$-quotient and $g$-index) were calculated (within the reviewed papers); the $h$-index is a metric that ranks the impact of an author through the frequency and citation count of the most-cited publications. A country collaboration network was created from documents with two or more authors affiliated with institutions in different countries.

\section{RESULTS}

The results of this study show a timeline similar to descriptions of the new, post-2006 wave of CT presented in research literature elsewhere (e.g., [19, 27]). The phrase "computational thinking” entered the common computing education parlance through Wing's 2006 essay in ACM's premier magazine Communications of the ACM [68]. Initially, the number of CT-related publications grew slowly: less than $13 \%$ of all articles in the dataset were published in the first half of the studied period (2006-2012). Over that period of time, there were concerted and well-funded efforts to bring CT into the everyday consciousness and vocabulary in computing fields as well as outside computing. For instance, in the United States, Wing successfully leveraged her position as the 2007-2010 head of Computer and Information Science and Engineering of the US National Science Foundation (NSF), which stated that winning CPATH funding from NSF required projects to demonstrate how CT is incorporated within the project [4, pp. 46 and 111; 30]. In 2008, members of Computer and Information Science and Engineering (NSF) asked the US National Research Council to organize two workshops on CT: one for discussing what the newly introduced phrase meant for the workshop participants [45, p. 7], and one for discussing educational and pedagogical questions related to CT [46]. Those workshops gathered together many computing pioneers and leading figures in computing education. The two workshop reports paint an excellent image of the challenges in reaching consensus over CT and ultimately over computing's disciplinary identity, and the reports reflect well the next decade of $\mathrm{CT}$ discussions.

Despite the lack of consensus over what CT is, the efforts started to bear fruit. The growth of CTrelated publications accelerated after 2012, and $87 \%$ of the titles were published in the second half of the study period, between 2013 and 2019. The annual output of CT-related articles accelerated rapidly over the second half, reaching 430 articles in 2019 with an annual percentage growth rate 


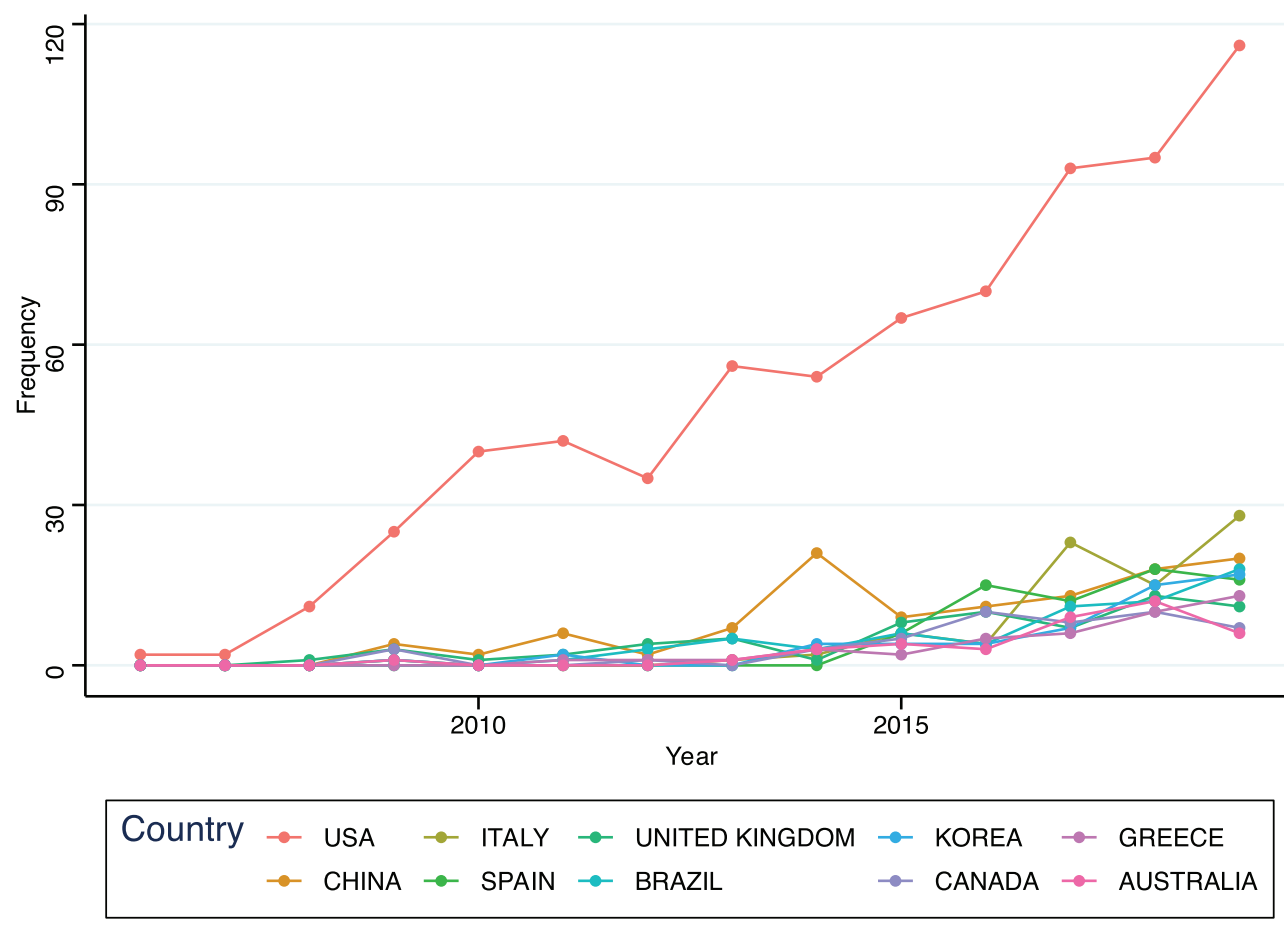

Fig. 1. The annual number of publications on CT over the period 2006-2019 in the most productive countries.

of $61.2 \%$. Figure 1 illustrates the trends of annual publishing activity on CT in the most productive countries.

The types of the most-cited publications on CT (Table 2) resemble the types of CER literature in general (cf. [58]), and the list in Table 2 includes all of the main types of CER literature: essays, reviews, position papers, and empirical research reports. Around two thirds of the publications $(68.7 \%)$ were published as conference papers, roughly a quarter $(25.2 \%)$ were journal articles, and approximately $6 \%$ were book chapters, editorials, and review papers. The most common identifiable source title was the Lecture Notes in Computer Science series ( 80 publications), followed by proceedings of the Frontiers in Education conference ( 75 publications) and proceedings of the ASEE Annual Conference (54 publications).

The most influential CT publication in the Scopus dataset, with 1,782 citations, was Wing's 2006 essay [68] that introduced the modern meaning of the phrase "computational thinking," and that started the CT bandwagon rolling. Wing wrote her essay at a time when enrollments in computing were seriously declining, wanting to make computing a more inspiring career choice for the youth [47, p. 86]. The timing of Wing's article was opportune, and she was able to articulate the needs of the 21 st century in a way that resonated well with what many educators had witnessed themselves, too [60].

The top-cited contributions to CT became popular for different reasons, and they served different purposes. The second highest cited paper was a 2013 review and analysis of CT by Grover and Pea [25] that was published in a prominent education journal, Educational Researcher. At a time when $\mathrm{CT}$ was already gathering considerable interest, the paper became popular for successfully explaining to the journal's primary readership-educators-what CT is, why it had rapidly gathered so much interest, and what were the currently pertinent and upcoming research foci around 
Table 2. Top 15 Most Cited CT Publications in the Scopus Database

\begin{tabular}{llcc}
\hline Authors & \multicolumn{1}{c}{ Source Title } & GCS & LCS \\
\hline 68 & Communications of the ACM & 1,782 & 935 \\
25 & Educational Researcher & 499 & 168 \\
69 & Philosophical Transactions of the Royal Society A & 395 & 239 \\
40 & Computers in Human Behavior & 249 & - \\
9 & Computers \& Education & 178 & 81 \\
66 & Journal of Science Education and Technology & 158 & 105 \\
55 & Education and Information Technologies & 146 & 86 \\
26 & Communications of the ACM & 143 & - \\
50 & Proceedings of ACM SIGCSE '10 & 115 & 73 \\
67 & Proceedings of ACM SIGCSE '12 & 113 & 78 \\
14 & Communications of the ACM & 96 & 14 \\
52 & Computers \& Education & 90 & - \\
70 & ACM Transactions on Computing Education & 86 & 68 \\
64 & Education and Information Technologies & 81 & 57 \\
28 & Journalism & 69 & - \\
\hline
\end{tabular}

GCS, Global Citation Score; LCS, Local Citation Score.

the topic. The third highest cited paper was Wing's 2008 position paper [69] that responded to the dire need for clarifying what CT is. In her paper, which was presented to the 2008 British Royal Society discussion meeting, Wing elaborated on her earlier description of CT that was gradually gaining momentum.

The fourth most cited paper addressed the debate concerning the relationship between programming and CT in K-12 education. That paper was a 2014 review of empirical research on learning CT through programming, by Lye and Koh [40], and published in Computers in Human Behavior. Many organizations and authors had skirted around the subject of the role of programming in learning CT to an extent that was later criticized for "losing of the original definition of CT" [36], whereas people seldom recognized that for many authors, the goal of CT was "to bring programming back into the classroom" [31], which Lye and Koh's review made clear. Their review was widely read, and it was followed by years of lively debates about the role and relevance of programming in CT (e.g., [3, 10, 31, 35, 39, 44, 57]). Both Grover and Pea's 2013 [25] and Lye and Koh's 2014 [40] reviews noted an abundance of discussions over definitions of CT and urged CT researchers for more empirical, intervention-based research.

The full list of the top 15 most cited papers (Table 2) contains essays and viewpoints on CT $[14,26,68]$, theoretical contributions or position papers that define CT or add to it in one way or another $[28,50,64,66,69]$, literature reviews [25, 40], and empirical papers reporting on CT initiatives $[9,52,55,67,70]$. Many of the top-cited works gathered considerable interest also outside the fields of computing and CER, as witnessed by their high GCSs (Table 2). But what caught the attention outside computing education circles was slightly different from what became popular within CT circles: A number of articles with a high LCS (citations within the examined dataset) had a relatively low GCS; examples include the 2011 article by Lee et al. [37] in the computing education magazine ACM Inroads (LCS = 114), the description of CT by Aho [1] (LCS = 55), the 2009 position paper on CT and programming by Lu and Fletcher [39] (LCS = 47), the CT course description of Hambrusch et al. [29], and the assessment framework of Seiter and Foreman [54].

Many influential CT publications have cited relatively little other CT literature. A historical direct citation network (Figure 2) shows the relationships between CT articles that have been 


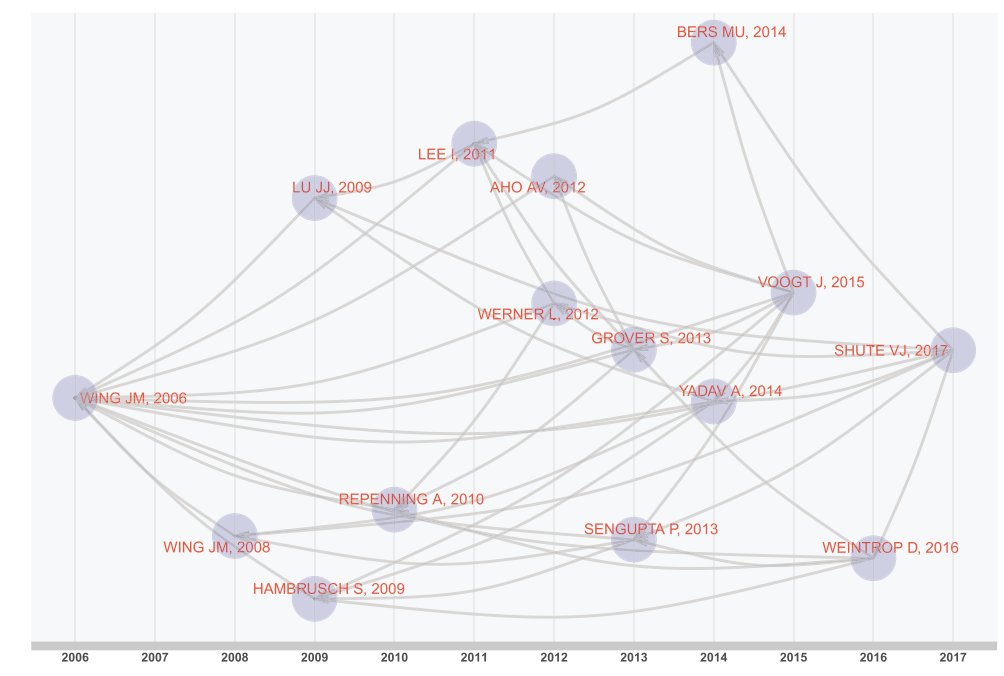

Fig. 2. A historical direct citation network of the 15 most influential papers within the field (highest LCSs).

Table 3. Keyword Frequencies in CT Literature

\begin{tabular}{lccccccc}
\hline \multicolumn{2}{c}{ Author Keywords } & \multicolumn{2}{c}{ Categorized Keywords } & \multicolumn{2}{c}{ Occurrences in Title } & \multicolumn{2}{c}{ Occurrences in Abstract } \\
Keyword & $N$ & Keyword & $N$ & Keyword & $N$ & Keyword & $N$ \\
\hline CT & 1,142 & CT & 1,155 & CT & 897 & CT & 4,207 \\
Programming & 147 & Programming & 510 & Programming & 312 & Students & 3,381 \\
CSE & 113 & Education & 359 & Learning & 296 & Programming & 2,012 \\
Education & 93 & STEM & 137 & Education & 229 & Learning & 1,918 \\
Scratch & 87 & Games & 116 & Students & 188 & Education & 1,323 \\
Problem-solving & 75 & K12 & 108 & Teaching & 181 & Skills & 1,272 \\
Coding & 63 & CS & 84 & School & 164 & Study & 1,016 \\
STEM & 57 & Robotics & 79 & CS & 135 & Computing & 1,012 \\
Assessment & 53 & Problem-solving & 75 & Skills & 120 & CS & 972 \\
K12 & 51 & Assessment & 53 & Computational & 118 & Teaching & 942 \\
\hline
\end{tabular}

The latter two analyses (title and abstract) count each occurrence within the title and abstract.

CT, Computational Thinking; CSE, Computer Science Education; CS, Computer Science.

central for defining the topic. The citation network of influential papers within the topic (highest LCSs) in Figure 2 shows that those papers have exerted only limited influence on each other. The number of literature references in the articles in Figure 2 varies greatly from roughly a dozen in many articles to nearly a hundred or more $[55,66]$.

\subsection{Keyword Analysis}

Based on the keyword sections of CT papers, programming and $\mathrm{K}-12$ education were the dominant themes in CT studies. The author-selected keyword "computational thinking" and its variants (e.g., CT skills and CT patterns) appeared in the keywords section of 1,155 (61.6\%) papers. Programming- and coding-related terms were the next most frequent, appearing in $510(27.2 \%)$ publications. Education-related terms, such as teaching, learning, and pedagogy, appeared in 359 (19.1\%) papers. Table 3 shows the top 10 keywords under keywords given by the authors, categorized keywords, keywords extracted from the article titles, and keywords extracted from the 


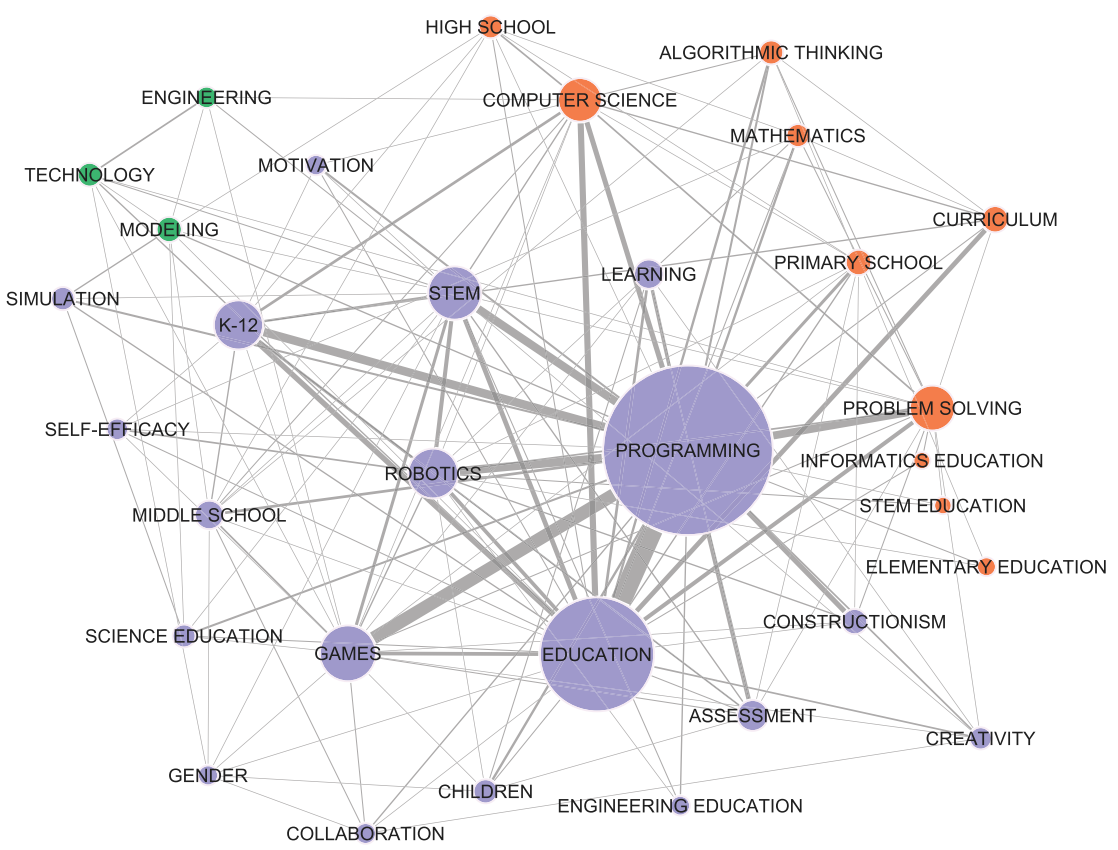

Fig. 3. Associations between keywords in the dataset, with the keyword "computational thinking" removed. (Circle size indicates keyword count in the sample, line thickness indicates co-occurrence frequency, and colors indicate a "community" or cluster of keywords).

abstracts. The last two (occurrences in title and occurrences in abstract) count each occurrence separately: For instance, if an abstract mentions the keyword "programming" three times, that counts as three occurrences.

In keyword association analysis, three keywords-"CT," "programming," and "education"formed a triangle, signifying a strong association between the themes. Figure 3 uses keyword association networks to visualize co-occurrences between keywords. The network in Figure 3 demonstrates the conceptual structure in the analyzed set of CT papers and the relational dimensions between concepts in that dataset. That keyword association network was constructed from the categorized keyword list where the keyword "computational thinking" was removed (it did most often co-occur with the keywords "programming" and "education").

After removing the "CT" keyword for readability, the co-occurrence network in Figure 3 sheds light on the nature of $\mathrm{CT}$ as a distinctively $\mathrm{K}-12$ educational initiative. A dense cluster of school concepts formed around two key concepts- "programming" and "education"- along with its curricular integration "keyword", "STEM." Those three were surrounded by the keywords K-12, children, and higher education, as well as primary, middle, and high school. Computing's traditional disciplinary dimensions [17] were all present: design (through keywords like creativity, robotics, technology, and engineering), modeling (simulation, abstraction, STEM), and theory (algorithm/algorithmic thinking, problem-solving, computation/computing, informatics). The education side of CER featured strongly in keywords like constructionism, self-efficacy, assessment, motivation, teacher training, games, and collaboration, as well as gender.

\subsection{Author Analysis}

Whereas Wing's 2006 essay [68] holds top position in terms of citations, other researchers have picked up top positions in terms of number of CT publications. Table 4 shows the 15 most active 
Table 4. Most Active Contributors to the CT Body of Knowledge, Sorted by Number of Scopus-Indexed Papers Published

\begin{tabular}{lccccccc}
\hline$\#$ & Author & Aff. & $N$ & $h$ & Earliest & Cites & Most Cited Paper \\
\hline 1 & Repenning A & US & 33 & 13 & 2009 & 544 & 50 \\
2 & Biswas G. & US & 23 & 7 & 2012 & 276 & 55 \\
3 & Basu S. & US & 20 & 7 & 2012 & 285 & 55 \\
& Robles G & ES & 20 & 10 & 2015 & 208 & 43 \\
5 & Fronza I & IT & 17 & 3 & 2015 & 52 & 20 \\
& Grover S & US & 17 & 6 & 2013 & 688 & 25 \\
7 & Moreno-León J & ES & 16 & 9 & 2015 & 193 & 43 \\
& Sengupta P & US & 16 & 7 & 2012 & 257 & 55 \\
9 & Dagienė V & LT & 14 & 7 & 2011 & 164 & 41 \\
& Koh KH & US & 14 & 10 & 2009 & 320 & 33 \\
& Soh LK & US & 14 & 6 & 2009 & 135 & 56 \\
12 & Basawapatna A & US & 13 & 9 & 2009 & 255 & 7 \\
& García-Peñalvo FJ & ES & 13 & 6 & 2016 & 124 & 21 \\
& Wilensky U & US & 13 & 7 & 2014 & 238 & 66 \\
15 & Settle A & US & 12 & 6 & 2009 & 186 & 41 \\
& Shell DF & US & 12 & 6 & 2009 & 135 & 56 \\
& Wiebe E & US & 12 & 2 & 2008 & 21 & 42 \\
\hline
\end{tabular}

Aff., country of affiliation in the most-cited article; $N$, number of articles; $h, h$-index.

contributors to CT in terms of number of papers (there were several authors with 12 publications, who all are listed, making the list 17 names long). The list of authors is very US-centric: 12 people on the list of 17 authors were affiliated with US-based institutions at the time of their most-cited publication. All of the most productive CT authors in Table 4 have published 12 or more Scopusindexed papers on CT. Table 4 presents the most active authors, the number of articles they have co-authored, their $h$-indexes within this dataset, the year of their first CT-related entry in Scopus, the total number of citations to their CT-related articles, and their most-cited papers.

The list of most active authors on CT by volume (Table 4) partly overlaps with the list of mostcited CT publications (Table 2), but the differences between those tables show that influence is not necessarily about volume. Some of the most influential papers were written by people with only a few CT-related publications. Examples include Wing, whose two essays [68, 69] hold top positions in terms of citations but who has since published rarely on the topic; Bers et al. [9], famous for their construction-based robotics activities around CT; Guzdial [26], who has actively advocated strengthening the link between CT initiatives and existing work in the field of CER; and Werner et al. [67] with their work on measuring CT. Other examples include Denning [14], who has been the voice of computing as a discipline for decades (e.g., $[17,18]$ ) but whose major contributions to computing's disciplinary self-understanding have been framed in traditional computing vocabulary, and only some of them have used CT vocabulary (e.g., [14, 15, 19]). It is noteworthy that some of the most prolific authors only became involved in CT in 2015 or 2016: Figure 4 shows a timeline of publishing activity of the most productive CT authors). Furthermore, whereas each of the authors in Table 4 has gathered recognition in the field with some well-cited papers on CT, the $h$-indexes in Table 4 show that much work on CT also gets ignored. For instance, $h$-index 3 means that the author has three CT papers cited at least three times, and $h$-index 4 would require four of the author's CT papers each cited at least four times.

As the list of most active contributors to CT suggests, almost all of those authors belong to active research teams or researcher networks focused on CT-related topics. Many authors in Table 4 share 


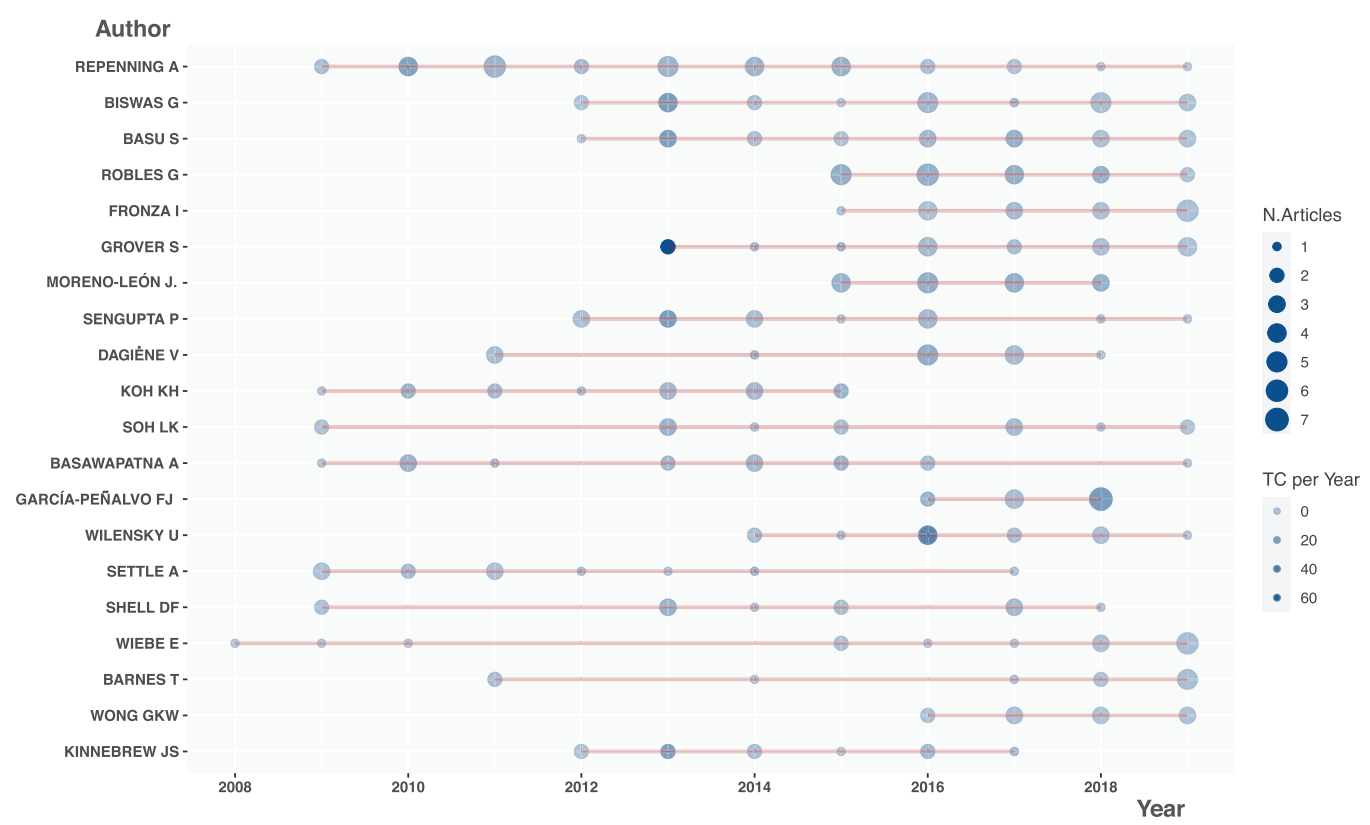

Fig. 4. Timeline of publishing activity of the most productive CT authors (circle size indicates the number of articles each year, and circle color indicates citations to that person's articles published in that year).

the same most-cited paper, and two especially influential clusters emerge from author network analysis, shown in Figure 5: A relatively tight network with A. Repenning as the most active paper author (e.g., [34, 50]), and a larger network where S. Basu and S. Biswas have been the most active paper authors in their co-author network (e.g., [55]). Figure 5 shows co-authorships between the 50 most active $\mathrm{CT}$ authors by the number of publications.

\subsection{Country Analysis}

Research output was also analyzed at the country scale to give an overview of geographical spread of CT research activity. The dataset was analyzed for the country of the first author, who typically (but not always) is considered the lead author of a study (however, as the number of multi-country publications was small compared to the number of single-country publications, the results are roughly in the right order of magnitude even if that assumption does not hold). By that measure, the dataset contained papers with lead authors from 64 countries. The article metadata were then analyzed for the countries of the rest of the authors. Table 5 ranks the 10 most active countries by the number of papers from each country, and reports the number of papers, the percentage of papers from each country, the number of single-country and multiple-country publications, the number of citations to papers from that country, and the mean citations per paper for each country.

The United States dominates CT-related research output-although inclusion of publications in other languages (which were excluded from this study) could slightly change that balance. NSF's CT-focused efforts [4] seem to have worked, as $42 \%$ of all CT-related articles had a US-based lead author, a great majority of those were single-country publications (only US-based institutions were involved), and 9 out of 15 most influential CT papers in Figure 2 thanked NSF support in their acknowledgments section. The next nine countries combined produced fewer CT publications than the United States did. That difference is not explainable by larger overall research output of the United States: The proportion of US research output in CT-related topics is more than twice the 


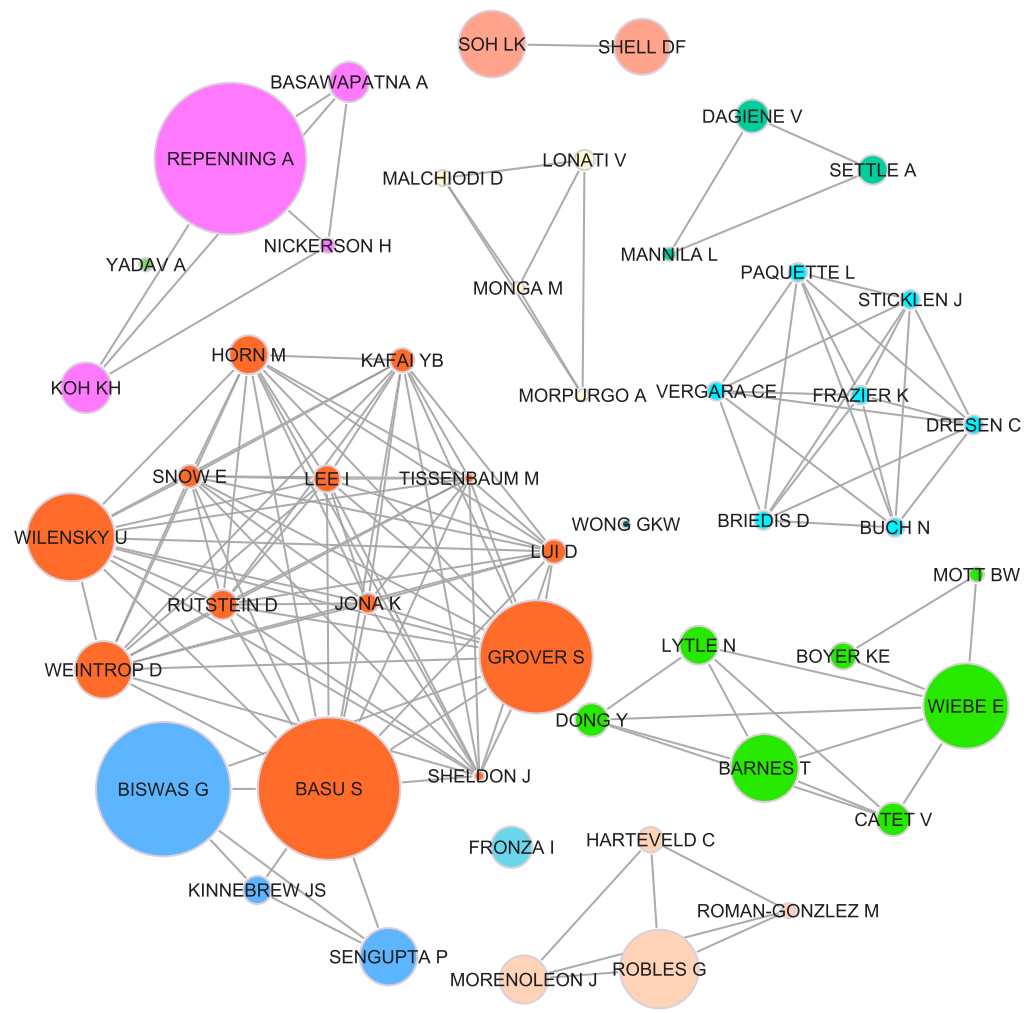

Fig. 5. Co-author network of CT-related articles in the Scopus database circle size indicates paper authorship, and colors indicate distinct communities of researchers who frequently collaborate together).

Table 5. Publications by the First Author's Country (2006-2019)

\begin{tabular}{lcccccc}
\hline Country & Papers & Freq. & SCP & MCP & Cites & Mean Cites \\
\hline United States & 556 & $41.65 \%$ & 521 & 35 & 7,771 & 13.98 \\
China & 81 & $6.07 \%$ & 71 & 10 & 142 & 1.75 \\
Spain & 64 & $4.79 \%$ & 48 & 16 & 399 & 6.23 \\
Italy & 59 & $4.42 \%$ & 45 & 14 & 173 & 2.93 \\
South Korea & 53 & $3.97 \%$ & 53 & 0 & 43 & 0.81 \\
United Kingdom & 47 & $3.52 \%$ & 36 & 11 & 321 & 6.83 \\
Brazil & 46 & $3.45 \%$ & 37 & 9 & 108 & 2.35 \\
India & 26 & $1.95 \%$ & 25 & 1 & 71 & 2.73 \\
Germany & 25 & $1.87 \%$ & 20 & 5 & 39 & 1.56 \\
Canada & 22 & $1.65 \%$ & 16 & 6 & 111 & 5.05 \\
\hline
\end{tabular}

SCP, single-country publication; MCP, multiple-country publication.

proportion of US research output in all fields (17.3\% of world research output) and three times that of US research output in CS (13.4\% of the world CS output) (2018 Scopus data). US-based publications also gathered many more citations $(7,771)$ than the others did $(1,407$ citations between the nine countries in Table 5) with a massive lead in mean citations per publication (nearly 14 citations per article). Although many prominent CT-related initiatives, such as Dagienè's Bebras 


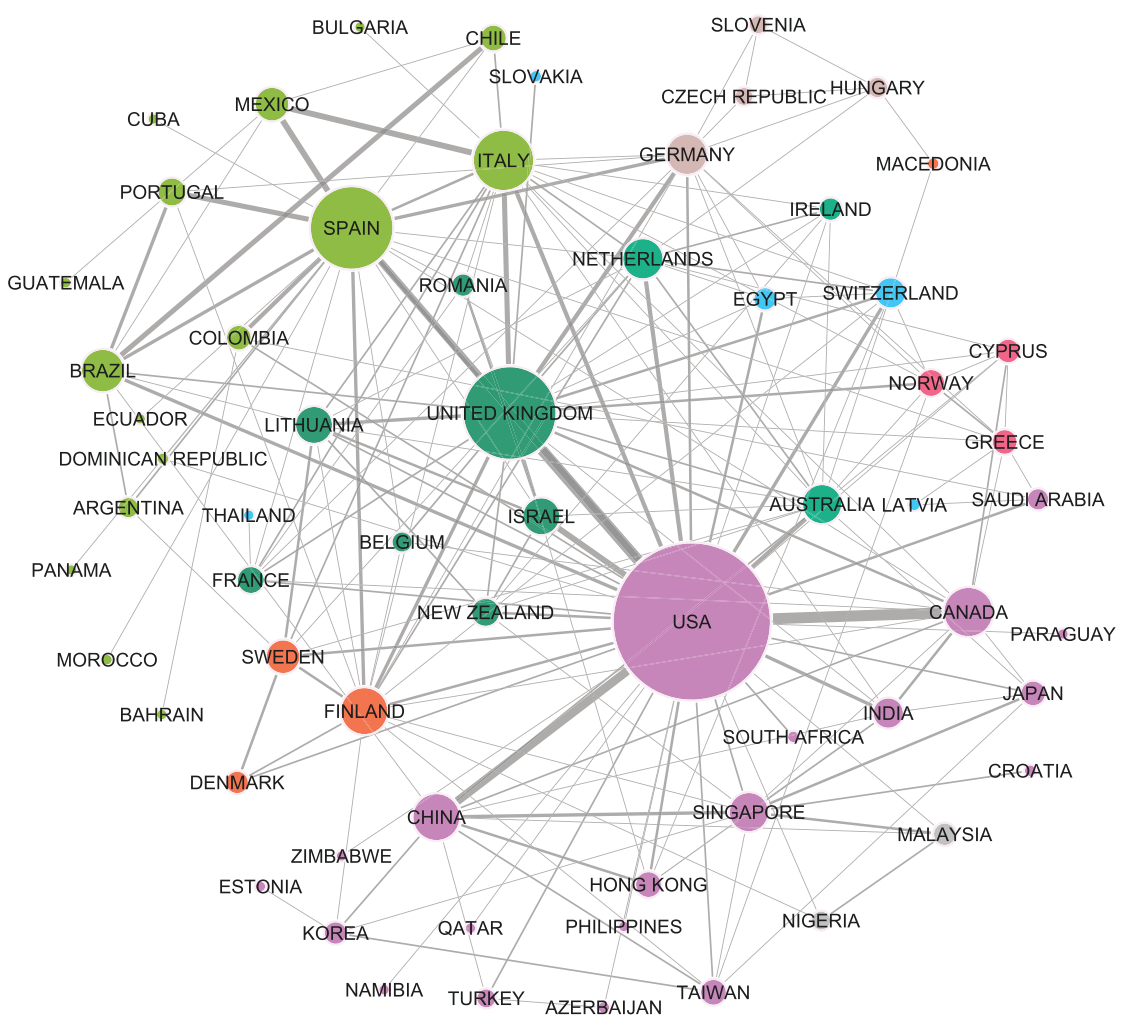

Fig. 6. Network of international collaborations in CT-related articles (countries that frequently collaborate have a similar color; circle size indicates papers per country, and line thickness indicates the number of co-authored articles).

Challenge [12] and Bell's CS Unplugged [8] come from outside the United STates, the bulk of CT research comes from US-based initiatives and institutions, citing mostly US-based research.

$\mathrm{CT}$ publications are distinctively national not only in the United States but also often elsewhere, with limited international collaboration-although that varies greatly between countries. In some countries, one in four or five publications were done with international collaboration; the most international studies were published by lead authors from Spain (where $25.0 \%$ of papers were multiple-country publications), Italy (23.7\%), the UK (23.4\%), and Brazil (19.6\%). Papers with lead researchers from the United States (6.3\%), India (3.9\%), and South Korea (0\%) had notably less international collaboration. CT research activity has rapidly grown in some of the countries included, and the most productive countries (the United States excluded) produced between $70 \%$ and $97 \%$ of their CT research output in 2018 and 2019. One perspective to the disproportionate amount of USbased research on CT is that by the time other countries jumped on the CT bandwagon, US-based researchers had already produced hundreds of papers on the topic.

Network analysis of international collaboration networks, based on countries of all paper authors, revealed large and diverse collaboration groups that still followed some regional and linguistic divisions. Figure 6 shows a network analysis of country-level collaborations: In the figure, circle size indicates the number of papers per country, line thickness indicates the number of coauthored articles, and circle color indicates the clusters of countries from which authors frequently collaborate. The United States had the most collaborating country partners (38), followed by the UK (24), Spain (23), Italy (19), Finland (17), and Australia (15). 
Countries were clustered using community detection, which showed Spain, Italy, and Portugal forming one community with the South American countries of Chile, Mexico, Brazil, Colombia, and Argentina. Another cluster was formed by the three Nordic countries of Sweden, Finland, and Denmark, and another by the Czech Republic, Hungary, Slovenia, and Germany. The largest cluster was formed around the United States and Canada with a number of Asian and Middle Eastern countries around it. Despite the relatively national nature of CT publications in the United States, the United States still had the highest betweenness centrality, highlighting its importance in bridging links between other countries; Spain scored second on that measure.

\section{DISCUSSION}

CT literature inherits some characteristics from the broader computing literature. Two of those characteristics-legitimate venues for publishing and types of research published-have been a source of lively debates in computing. First, CT literature inherits field-specific publishing preferences from computing as a discipline, which has traditionally focused on conferences stronger than other fields do [65]. With two thirds of the publications, conference papers were the most common form of publishing CT literature, and one in four publications were journal articles. But journal articles were cited much more (mean 10.5 citations per journal article) than conference papers (mean 3.8 citations per paper)-surpassed only by review articles (mean 41.9 citations per article). Second, CT literature inherits the diversity of paper types from CER, where researchers have for more than three decades discussed the need for more empirical research, and where the shift toward empirical research has been considered a sign of maturing of the field [58]. CER literature has traditionally been a mixture of position papers, essays, course reports, empirical research, literature reviews, and systems papers (e.g., see [58]), and the CT body of literature shares that characteristic, as witnessed by the diverse nature of CT's most-cited publications.

$\mathrm{CT}$ research lacks paradigmatic exemplars and strong consensus on methods, theories, and what significant results look like. A set of influential CT articles can clearly be identified, but instead of forming a grand narrative, they are influential for very different reasons and are intended for very different audiences: Some became influential for their persuasive argument, some for reporting innovative CT initiatives, and some for explaining CT to audiences outside computing. In terms of citing patterns, the key influential articles in the first half of the studied period (Figure 2, 20062012) cited other CT entries sparsely, and their lists of references were short in general, too (their bibliographies contained 13.6 mean cited items per article). In the second half of the studied period, the CT research community saw an increased consensus of significant CT literature, and the period saw many more citations to CT-specific literature and much longer lists of references-also in articles that were not literature reviews.

The most influential authors were not necessarily the most prolific: There are only a handful of people who are both on the list of authors of most-cited articles in CT and at the same time on the list of most active contributors to $\mathrm{CT}$ in terms of publication numbers. But although some opinion leaders' contributions to CT and CER body of literature are limited to a small number of position papers, the same people can at the same time be known for their influential, massive scale CT advocacy work with transformative impact.

$\mathrm{CT}$ has been a relatively US-centric movement from the start. The first, slowly growing wave of CT publications (2006-2012) (Figure 1) was heavily United States based. The US institutions dominated the second wave, too (2013-2019), but institutions from a growing number of countries joined the second wave. Still, 12 of the 17 most prolific CT authors were affiliated with US-based institutions in their most-cited publications, $42 \%$ of all $\mathrm{CT}$ papers had lead authors from US-based institutions, and US-led publications were cited over five times more than the next nine countries combined. NSF's role in the early years of CT cannot be downplayed: By requiring all 
CPATH-funded projects to address the term "computational thinking," NSF funding ensured that some of the leading CER groups in the United States framed their work in CT vocabulary. Funding from the NSF was acknowledged in 9 out of 15 most influential papers in Figure 2.

Other countries started to join the movement in the second half of the investigation period (2013-2019), but the United States remains by far the largest producer of CT-related research (Figure 1), with a disproportionately high share of papers compared to the country's share of research in CS as well as in all fields of research. The United States has some multi-country publications (6.3\%), but CT research in many countries is much more international than US-based research is. The expansion of CT research outside the United States alleviates risks such as cultural bias, diminished applicability, and losing richness of ideas and approaches from other educational and intellectual contexts. There are relatively active clusters of international collaboration centered around, for example, Spain, Italy, and Brazil, but their absolute number of multi-country publications is relatively small-around 10 or so per country. There may be various reasons for the very low citation numbers to South Korean (0.81 mean cites) and Chinese (1.75 mean cites) CT publications, such as the differences between countries' educational systems that complicates collaboration as well as applicability of many K-12 CT projects. Very little CT research originated in Africa, a home to 1.2 billion people, although as this study was limited to publications in English, any major publishing outlets in other languages were not accounted for. To what extent a predominantly western body of K-12 CT literature can cater to the needs of education systems on all continents and all countries remains an open question.

Kafai [31] wrote that CT is about programming-a "battle cry for coding in $\mathrm{K}-12$ education," yet the role of programming in $\mathrm{K}-12 \mathrm{CT}$ was heavily debated in the beginning. These results confirm Kafai's view, and they also confirm that much of the CT literature is about "CT for beginners" and how to teach programming-related concepts to children [19]. Keyword analysis and keyword co-occurrence network analysis associated CT is strongest with programming-related keywords and with a variety of education-related keywords, each of which point at distinct branches of CTrelated research (e.g., K-12, STEM, assessment, and robotics). Assessment, which has often been identified as a main weakness of CT initiatives [16], is among the top 10 keywords in CT publications. The keyword co-occurrence network further featured elements of computing's traditional tripartite disciplinary structure-design, modeling, and theory-and it also showed a number of prominent pedagogical and educational features of CT, including constructionism, self-efficacy, motivation, and teacher training, for example.

As CT continues to develop as a research topic, it faces pressure to keep aligned with and connected to CER and its prevailing trends, including developing stronger theoretical grounding and empirically established knowledge base. What remains to be seen is to what extent the ongoing extension of computing from the classical "good old rule-driven programming" to data-driven automation changes CT and CER. There is a growing recognition of the need to better understand how people, including children in K-12 education, learn artificial intelligence and machine learning, yet that shift is not yet well visible in CT literature. And to fulfill its original promise to better prepare learners for a world driven by algorithms, there still is work to be done on extending CT beyond the currently popular groups-K-12 learners and CS students-into educating people like doctors, economists, lawyers, and all other groups whose jobs are likely to be affected [53].

\section{REFERENCES}

[1] Alfred V. Aho. 2011. Ubiquity symposium: Computation and Computational Thinking. Ubiquity 2011 (2011), Article 1.

[2] Massimo Aria and Corrado Cuccurullo. 2017. Bibliometrix: An R-tool for comprehensive science mapping analysis. Journal of Informetrics 11, 4 (2017), 959-975. 
[3] Michal Armoni. 2016. Computer science, computational thinking, programming, coding: The anomalies of transitivity in K-12 computer science education. ACM Inroads 7, 4 (2016), 24-27.

[4] William Aspray. 2016. Participation in Computing: The National Science Foundation's Expansionary Programs. Springer, Switzerland.

[5] Valerie Barr. 2016. Disciplinary thinking, computational doing: Promoting interdisciplinary computing while transforming computer science enrollments. ACM Inroads 7, 2 (2016), 48-57.

[6] Valerie Barr and Chris Stephenson. 2011. Bringing computational thinking to K-12: What is involved and what is the role of the computer science education community? ACM Inroads 2, 1 (2011), 48-54.

[7] Ashok Basawapatna, Kyu Han Koh, Alexander Repenning, David C. Webb, and Krista Sekeres Marshall. 2011. Recognizing computational thinking patterns. In Proceedings of the 42nd ACM Technical Symposium on Computer Science Education (SIGCSE '11). ACM, New York, NY, 245-250. DOI : https://doi.org/10.1145/1953163.1953241

[8] Tim Bell, Jason Alexander, Isaac Freeman, and Mick Grimley. 2009. Computer science unplugged: School students doing real computing without computers. New Zealand fournal of Applied Computing and Information Technology 13 1 (2009), 20-29.

[9] Marina Umaschi Bers, Louise Flannery, Elizabeth R. Kazakoff, and Amanda Sullivan. 2014. Computational thinking and tinkering: Exploration of an early childhood robotics curriculum. Computers \& Education 72 (2014), $145-157$.

[10] Richard Connor, Quintin Cutts, and Judy Robertson. 2017. Keeping the machinery in computing education. Communications of the ACM 60, 11 (2017), 26-28.

[11] Paul Curzon, Tim Bell, Jane Waite, and Mark Dorling. 2019. Computational thinking. In The Cambridge Handbook of Computing Education Research, Sally A. Fincher and Anthony V. Robins (Eds.). Cambridge University Press, 513-546.

[12] Valentina Dagiene and Sue Sentance. 2016. It's computational thinking! Bebras tasks in the curriculum. In Informatics in Schools: Improvement of Informatics Knowledge and Perception, Andrej Brodnik and Françoise Tort (Eds.). Springer International Publishing, Cham, Switzerland, 28-39.

[13] Pasquale De Meo, Emilio Ferrara, Giacomo Fiumara, and Alessandro Provetti. 2011. Generalized Louvain method for community detection in large networks. In Proceedings of the 11th International Conference on Intelligent Systems Design and Applications. 88-93.

[14] Peter J. Denning. 2009. Beyond computational thinking. Communications of the ACM 52, 6 (2009), 28-30.

[15] Peter J. Denning. 2017. Computational thinking in science. American Scientist 105, 1 (2017), 13-17.

[16] Peter J. Denning. 2017. Remaining trouble spots with computational thinking. Communications of the ACM 60, 6 (2017), 33-39.

[17] Peter J. Denning, D. E. Comer, David Gries, Michael C. Mulder, Allen Tucker, A. Joe Turner, and Paul R. Young. 1989. Computing as a discipline. Communications of the ACM 32, 1 (1989), 9-23.

[18] Peter J. Denning and Craig H. Martell. 2015. Great Principles of Computing. MIT Press, Cambridge, MA.

[19] Peter J. Denning and Matti Tedre. 2019. Computational Thinking. MIT Press, Cambridge, MA.

[20] Ilenia Fronza, Nabil El Ioini, and Luis Corral. 2015. Students want to create apps: Leveraging computational thinking to teach mobile software development. In Proceedings of the 16th Annual Conference on Information Technology Education (SIGITE'15). ACM, New York, NY, 21-26.

[21] F. J. García-Peñalvo. 2016. A brief introduction to TACCLE 3-Coding European project. In Proceedings of the 2016 International Symposium on Computers in Education (SIIE'16). 1-4. DOI : https://doi.org/10.1109/SIIE.2016.7751876

[22] Francisco Jose García-Peñalvo, Daniela Reimann, Maire Tuul, Angela Rees, and Ilkka Jormanainen. 2016. An Overview of the Most Relevant Literature on Coding and Computational Thinking with Emphasis on the Relevant Issues for Teachers. Technical Report. TACCLE3 Consortium, Belgium. DOI : https://doi.org/doi:10.5281/zenodo.165123

[23] Eugene Garfield. 2004. Historiographic mapping of knowledge domains literature. fournal of Information Science 30, 2 (2004), 119-145.

[24] Shuchi Grover. 2018. A tale of two CTs (and a revised timeline for computational thinking). Blog@CACM (November 5, 2018). Retrieved January 23, 2021 from https://cacm.acm.org/blogs/blog-cacm/232488-a-tale-of-two-cts-anda-revised-timeline-for-computational-thinking/fulltext.

[25] Shuchi Grover and Roy D. Pea. 2013. Computational thinking in K-12: A review of the state of the field. Educational Researcher 42, 1 (2013), 38-43.

[26] Mark Guzdial. 2008. Education: Paving the way for computational thinking. Communications of the ACM 51, 8 (2008), $25-27$.

[27] Mark Guzdial. 2015. Learner-Centered Design of Computing Education: Research on Computing for Everyone. Morgan \& Claypool, San Rafael, CA.

[28] Astrid Gynnild. 2014. Journalism innovation leads to innovation journalism: The impact of computational exploration on changing mindsets. Journalism 15, 6 (2014), 713-730.

[29] Susanne Hambrusch, Christoph Hoffmann, John T. Korb, Mark Haugan, and Antony L. Hosking. 2009. A multidisciplinary approach towards computational thinking for science majors. In Proceedings of the 40th ACM Technical Symposium on Computer Science Education (SIGCSE’09). ACM, New York, NY, 183-187. 
[30] David Hemmendinger. 2010. A plea for modesty. ACM Inroads 1, 2 (2010), 4-7.

[31] Yasmin B. Kafai. 2016. From computational thinking to computational participation in K-12 education. Communications of the ACM 59, 8 (2016), 26-27.

[32] Donald E. Knuth. 1974. Computer science and its relation to mathematics. American Mathematical Monthly 81 (1974), 323-343.

[33] Kyu Han Koh, Ashok Basawapatna, Vicki Bennett, and Alexander Repenning. 2010. Towards the automatic recognition of computational thinking for adaptive visual language learning. In Proceedings of the 2010 IEEE Symposium on Visual Languages and Human-Centric Computing. 59-66.

[34] K. H. Koh, A. Basawapatna, H. Nickerson, and A. Repenning. 2014. Real time assessment of computational thinking. In Proceedings of the 2014 IEEE Symposium on Visual Languages and Human-Centric Computing (VL/HCC'14). 49-52.

[35] Anna Lamprou and Alexander Repenning. 2017. Computational thinking $\neq$ programming. SI Magazine. Retrieved January 23, 2021 from https://magazine.swissinformatics.org/en/computational-thinking-\%e2\%89\%a0-programming/.

[36] Irene Lee. 2016. Reclaiming the roots of CT. CSTA Voice: The Voice of K-12 Computer Science Education and Its Educators 12, 1 (2016), 3-4.

[37] Irene Lee, Fred Martin, Jill Denner, Bob Coulter, Walter Allan, Jeri Erickson, Joyce Malyn-Smith, and Linda Werner. 2011. Computational thinking for youth in practice. ACM Inroads 2, 1 (2011), 32-37.

[38] James Lockwood and Aidan Mooney. 2017. Computational Thinking in Education: Where Does It Fit? A Systematic Literary Review. Technical Report. National University of Ireland Maynooth.

[39] James J. Lu and George H. L. Fletcher. 2009. Thinking about computational thinking. SIGCSE Bulletin 41, 1 (2009), $260-264$.

[40] Sze Yee Lye and Joyce Hwee Ling Koh. 2014. Review on teaching and learning of computational thinking through programming: What is next for K-12? Computers in Human Behavior 41 (2014), 51-61.

[41] Linda Mannila, Valentina Dagiene, Barbara Demo, Natasa Grgurina, Claudio Mirolo, Lennart Rolandsson, and Amber Settle. 2014. Computational thinking in K-9 education. In Proceedings of the Working Group Reports of the 2014 on Innovation and Technology in Computer Science Education Conference (ITiCSE-WGR'14). ACM, New York, NY, 1-29.

[42] Wookhee Min, Megan H. Frankosky, Bradford W. Mott, Jonathan P. Rowe, Eric Wiebe, Kristy Elizabeth Boyer, and James C. Lester. 2015. DeepStealth: Leveraging deep learning models for stealth assessment in game-based learning environments. In Artificial Intelligence in Education, Cristina Conati, Neil Heffernan, Antonija Mitrovic, and M. Felisa Verdejo (Eds.). Springer International Publishing, Cham, Switzerland, 277-286.

[43] Jesús Moreno-León and Gregorio Robles. 2015. Dr. Scratch: A web tool to automatically evaluate scratch projects. In Proceedings of the Workshop in Primary and Secondary Computing Education (WiPSCE'15). ACM, New York, NY, 132-133.

[44] Enrico Nardelli. 2019. Do we really need computational thinking? Communications of the ACM 62, 2 (2019), 32-35.

[45] National Research Council. 2010. Report of a Workshop on the Scope and Nature of Computational Thinking. National Academies Press, Washington, DC. DOI : https://doi.org/10.17226/12840

[46] National Research Council. 2011. Report of a Workshop on the Pedagogical Aspects of Computational Thinking. National Academies Press, Washington, DC. DOI : https://doi.org/10.17226/13170

[47] Sebastian Mateos Nicolajsen. 2020. Perspectives on Computational Thinking. IT University of Copenhagen, Copenhagen, Denmark.

[48] Seymour Papert. 1980. Mindstorms: Children, Computers, and Powerful Ideas. Basic Books, New York, NY.

[49] Arnold Neville Pears. 2019. Developing computational thinking, "Fad" or "Fundamental"? Constructivist Foundations 14, 3 (2019), 410-412.

[50] Alexander Repenning, David Webb, and Andri Ioannidou. 2010. Scalable game design and the development of a checklist for getting computational thinking into public schools. In Proceedings of the 41st ACM Technical Symposium on Computer Science Education (SIGCSE'10). ACM, New York, NY, 265-269.

[51] Mitchel Resnick. 2017. Lifelong Kindergarten: Cultivating Creativity Through Projects, Passion, Peers, and Play. MIT Press, Cambridge, MA.

[52] José-Manuel Sáez-López, Marcos Román-González, and Esteban Vázquez-Cano. 2016. Visual programming languages integrated across the curriculum in elementary school: A two year case study using "Scratch" in five schools. Computers \& Education 97 (2016), 129-141.

[53] Mohammed Saqr and Matti Tedre. 2019. Should we teach computational thinking and big data principles to medical students? International fournal of Health Sciences 13, 4 (2019), 1-2.

[54] Linda Seiter and Brendan Foreman. 2013. Modeling the learning progressions of computational thinking of primary grade students. In Proceedings of the 9th Annual International ACM Conference on International Computing Education Research (ICER'13). ACM, New York, NY, 59-66.

[55] Pratim Sengupta, John S. Kinnebrew, Satabdi Basu, Gautam Biswas, and Douglas Clark. 2013. Integrating computational thinking with K-12 science education using agent-based computation: A theoretical framework. Education and Information Technologies 18, 2 (2013), 351-380. 
[56] Duane F. Shell and Leen-Kiat Soh. 2013. Profiles of motivated self-regulation in college computer science courses: Differences in major versus required non-major courses. fournal of Science Education and Technology 22, 6 (2013), 899-913. DOI : https://doi.org/10.1007/s10956-013-9437-9

[57] Valerie J. Shute, Chen Sun, and Jodi Asbell-Clarke. 2017. Demystifying computational thinking. Educational Research Review 22 (2017), 142-158.

[58] Simon. 2015. Emergence of Computing Education as a Research Discipline. Ph.D. Dissertation. Aalto University, Finland.

[59] Cynthia J. Solomon. 1976. Teaching the Computer to Add: An Example of Problem-Solving in an Anthropomorphic Computer Culture. Memo 396. MIT AI Laboratory, Cambridge, MA.

[60] Matti Tedre and Peter J. Denning. 2016. The long quest for computational thinking. In Proceedings of the 16th Koli Calling International Conference on Computing Education Research (Koli Calling'16). ACM, New York, NY, 120-129.

[61] Josh Tenenberg. 2018. Computational making. ACM Inroads 9, 1 (2018), 22-23.

[62] Mike Tissenbaum, Josh Sheldon, and Hal Abelson. 2019. From computational thinking to computational action. Communications of the ACM 62, 3 (2019), 34-36.

[63] Annette Vee. 2017. Coding Literacy: How Computer Programming Is Changing Writing. MIT Press, Cambridge, MA.

[64] Joke Voogt, Petra Fisser, Jon Good, Punya Mishra, and Aman Yadav. 2015. Computational thinking in compulsory education: Towards an agenda for research and practice. Education and Information Technologies 20, 4 (2015), 715-728.

[65] George Vrettas and Mark Sanderson. 2015. Conferences versus journals in computer science. Fournal of the Association for Information Science and Technology 66, 12 (2015), 2674-2684. DOI : https://doi.org/10.1002/asi.23349

[66] David Weintrop, Elham Beheshti, Michael Horn, Kai Orton, Kemi Jona, Laura Trouille, and Uri Wilensky. 2016. Defining computational thinking for mathematics and science classrooms. Fournal of Science Education and Technology 25, 1 (2016), 127-147.

[67] Linda Werner, Jill Denner, Shannon Campe, and Damon Chizuru Kawamoto. 2012. The fairy performance assessment: Measuring computational thinking in middle school. In Proceedings of the 43rd ACM Technical Symposium on Computer Science Education (SIGCSE'12). ACM, New York, NY, 215-220.

[68] Jeannette M. Wing. 2006. Computational thinking. Communications of the ACM 49, 3 (2006), 33-35.

[69] Jeannette M. Wing. 2008. Computational thinking and thinking about computing. Philosophical Transactions of the Royal Society A 36, 1881 (2008), 3717-3725.

[70] Aman Yadav, Chris Mayfield, Ninger Zhou, Susanne Hambrusch, and John T. Korb. 2014. Computational thinking in elementary and secondary teacher education. ACM Transactions on Computing Education 14, 1 (2014), Article 5, 16 pages.

Received April 2020; revised November 2020; accepted December 2020 